\title{
Estimation of Dynamic Response Index Domain of High Speed Railway Vehicle System
}

\author{
Xulu WANG ${ }^{1}$ and Limin CHANG \\ School of Mechanical and Electrical Engineering, Lanzhou Jiaotong University \\ Lanzhou, China
}

\begin{abstract}
Based on the dynamic simulation model, the dynamic response index of vehicle system under the action of track irregularity is divided into three areas: repair, deterioration and maintenance. The correlation between the track irregularity index and the dynamic response index domain of vehicle system components is calculated and statistically studied. The estimation model of dynamic response index domain of vehicle system and the domain boundaries of different dynamic response indexes are established and obtained Line. According to the principle of single variable method, the excitation source of vehicle track system is divided into track irregularity and other comprehensive factors (such as temperature load, material damage, etc.), and a simple inversion method of track foundation state is proposed based on the estimation model of dynamic response index domain. Its basic principle is: if the statistical characteristics of track irregularity remain unchanged and other influencing factors change, the estimation domain and measurement domain of dynamic response index will produce grade jump, so as to determine whether the basic state of the line is normal. The simulation results show that the accuracy of domain estimation of dynamic response index of vehicle system is more than $80 \%$, and the accuracy of recognition is more than $70 \%$ for the damage condition of the line infrastructure, where the fastener is empty.
\end{abstract}

Keywords. Dynamic simulation; statistical learning; dynamic inversion; domain estimation

\section{Introduction}

Based on the principle of dynamic interaction between vehicle system and track structure [1], track irregularity is one of the key excitation sources of dynamic coupling in wheel rail contact, so the dynamic behavior of vehicle track system is closely related to track irregularity [2]. Theoretically, there must be a linear or nonlinear relationship between track irregularity and dynamic response of vehicle rail system According to the high-speed railway line design specifications, set the line geometric parameters under different curve radius conditions, establish a complete locomotive-line system space model, use the multi-body dynamics simulation software UM for simulation calculation, and analyze the differences in detail car body, wheel-rail interaction and track dynamic response under curve parameters [3].

${ }^{1}$ Xulu Wang, Corresponding author, School of Mechanical and Electrical Engineering, Lanzhou Jiaotong University Lanzhou, China 2652530637@qq.com 
Taking the vehicle system dynamic response as input vector in reference [4], the recognition rate of harmonic irregularity wavelength and amplitude based on time series prediction, neural network pattern recognition, BP neural network and radial basis function network is studied and compared, and the recognition rate and recognition effect of four identification methods are compared and analyzed. In reference [5], according to the high-speed railway line design specifications, the geometric parameters of the line under different curve radius conditions are set up, a complete space model of the locomotive vehicle line system is established, and the multi-body dynamics simulation software um is used for simulation calculation, and the dynamic response of the train body, wheel rail interaction and track under different curve parameters conditions are analyzed in detail. In reference [6], a hierarchical design and weight combination method for parameter optimization of vehicle track coupling system is proposed. Based on the optimization of low dynamic interaction of wheel rail interface, the parameters of vehicle rail system are divided into two groups according to the hierarchical design idea, and the dynamic behavior of the system under different parameters matching is investigated; in addition, the influence degree of system parameters in different design layers on the optimization objective is quantitatively analyzed by using the method of weight coefficient.

The research of this paper is mainly based on the vertical coupling dynamics of vehicle track, the support vector machine (SVM) theory and the least square fitting method, this paper establishes the relevant dynamic calculation model for the slab track of high-speed railway. The simulation calculation method is used to obtain the dynamic response time of vehicle system under different track irregularity characteristics, and the dynamic response of different parts of vehicle system is used The target of index domain prediction and line infrastructure damage identification is to optimize the extraction of track irregularity control indexes that affect the dynamic response of vehicle system, to build the model of track irregularity index vertical dynamic response index domain estimation of vehicle system and to study the inversion of line infrastructure state.

\section{Estimation Model of Dynamic Response Index Domain of Vehicle System}

The dynamic response index domain estimation model of vehicle system mainly aims at the domain estimation of dynamic response indexes (absolute mean value, standard deviation, absolute maximum value) of vehicle system components under different track irregularity characteristics, establishes the correlation between the track irregularity control indexes and the dynamic response index domain level of vehicle system, and realizes the fast estimation of the domain level of vehicle system dynamic response indexes Count.

See Figure 1 for the construction process of dynamic response index domain estimation model of vehicle system components. 


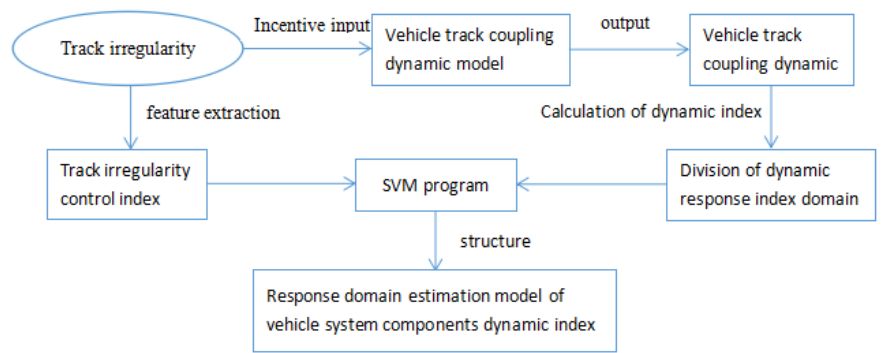

Figure 1. Construction process of dynamic response index domain estimation model of vehicle system components

The dynamic response index domain estimation model of vehicle system components is based on the extraction of track irregularity control index and the division of dynamic response index domain, and the relationship between them is established by SVM theory, thus forming the domain estimation model of vehicle system components dynamic response index. The following will respectively introduce the SVM theory, track irregularity control index extraction and dynamic response index domain estimation method.

\subsection{SVM Theory}

SVM is a supervised learning model, which is usually used for pattern recognition, classification and regression analysis. SVM is a machine learning method based on SRM and SLT [6]. In RN space, the essence of vector classification prediction problem is to find the function induced by learning samples, so that the function has better generalization. The principle is as follows $[7,8]$.

With the sample set $\left\{\left(x_{1}, y_{1}\right),\left(x_{2}, y_{2}\right), \cdots,\left(x_{n}, y_{n}\right)\right\}$ where $x_{i}=R^{n}(1,2 \cdots n)$ is the input mode, $y \in\{ \pm 1\}$ is the target output. Let the optimal plane be

$$
\Phi(\omega \xi)=\frac{1}{2} \omega^{T} \omega+C \sum_{i=1}^{n} \xi_{i}
$$

Where: $\xi_{i}$ is the relaxation variable, which indicates the deviation degree between the mode and the ideal linear situation; $\mathrm{C}$ is the positive parameter (penalty coefficient) to be specified, which indicates the penalty degree of SVM for the wrong samples. Then the weight vector $\omega$ and offset b must satisfy the following constraints

$$
y_{i}\left(\omega^{T} x_{i}+b\right) \geq 1-\xi_{i}
$$

The goal of SVM is to find a hyperplane which can minimize the average error classification of training data, so that the optimization problem can be deduced. According to the Lagrange multiplier method, the solution of the optimal classification hyperplane can be transformed into the following constrained optimization problems

$$
Q(a)=\sum_{i=1}^{n} \sum_{j=1}^{n} a_{i} a_{j} y_{i} y_{j} K\left(x_{i}, x_{j}\right)
$$




$$
\sum_{i=1}^{n} a_{i} y_{i}=0 \quad a_{i} \geq 0
$$

Where: $Q(a)$ is the objective function; $K\left(x_{i}, x_{j}\right)$ is the kernel function satisfying Mercer's theorem; $\left\{a_{i}\right\}^{n}$ is the Lagrangian multiplier, in which most of $a_{i}$ is 0 , and the samples corresponding to $a_{i}$ not equal to 0 are called support vectors, which can be used as the optimal classification surface of dynamic response index field.

\subsection{Track Irregularity Index for Domain Estimation}

The reasonable selection of the track irregularity index is the key factor in the domain estimation model of vehicle system dynamic response index, and it is also one of the problems that the domain estimation model is difficult to deal with. If the selection is not reasonable, it will not be able to correctly classify the different response areas of the dynamic indicators, resulting in more serious problems. In reference [7], the time-frequency statistical index of track irregularity is used to predict the vehicle body vibration response index. This method is used for reference in this paper. The commonly used track irregularity time-frequency statistical indicators are shown in Table 1.

Table 1. Time frequency statistical index of track irregularity

\begin{tabular}{ll}
\hline Time domain statistical index & Frequency domain statistical index \\
\hline Absolute mean value, maximum value, & Average frequency, peak standard deviation, \\
minimum value, square root mean value, peak value, peak frequency standard deviation, \\
square root amplitude, variance, peak peak maximum peak frequency, wavelet packet \\
value, skewness, kurtosis, peak index, subband energy (8-level decomposition of \\
waveform index, pulse index, margin index \\
and differential mean value
\end{tabular}

It can be seen from table 1 that the total number of time-frequency statistical indicators of track section irregularity is 275 (that is, 275 statistical indicators need to be calculated for each section irregularity sample). Generally, these indicators are redundant, and the most sensitive track irregularity control indicators to vehicle dynamic response can be obtained by using correlation coefficient [9]. The larger the correlation coefficient between the control index and the dynamic response index, the more obvious the excitation input-power output relationship between them. For 20166 samples, the distribution curve of the correlation coefficient between the absolute average value of the vertical vibration acceleration of the front frame and the track irregularity time-frequency statistical index is shown in Figure 2.

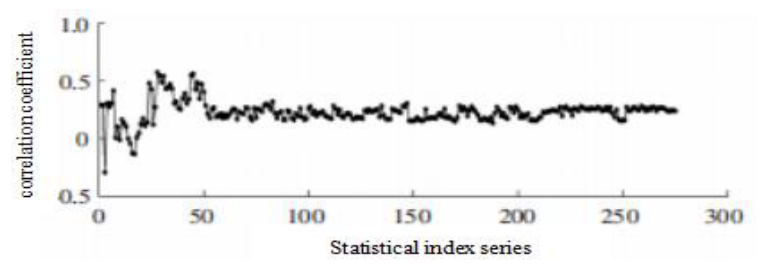

Figure 2. Distribution curve of correlation coefficient between absolute average value of vertical vibration acceleration of front frame and time-frequency statistical index of track irregularity 
It can be seen from Figure 2 that the correlation coefficient between the statistical indexes 28 and 45 and the absolute average index is the largest,0.5697 and 0.5593 respectively, which can be taken as two better control indexes. No.28 and No.45 statistical indexes are the 9th and 26th order subband energy of wavelet packet, and the calculation method can refer to reference [10].

\subsection{Domain Boundary Estimation Method of Dynamic Response Index}

The SVM method is used to calculate the optimal classification surface of the dynamic response index domain, extract the boundary coordinates between different index domains, and use the analytic function to fit the least square method to obtain the domain boundary of the vehicle system dynamic response index. See Figure. 6 for the distribution of track irregularity control indexes corresponding to different absolute average index fields of front frame acceleration.

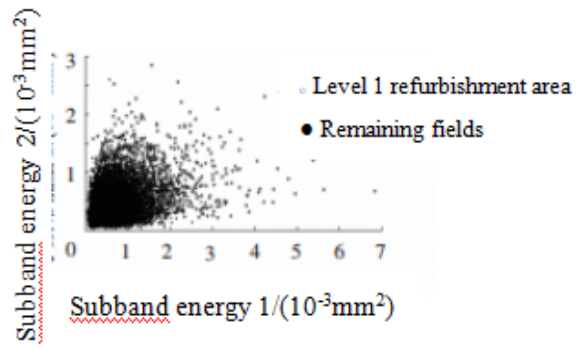

(a) Scatter distribution of control indexes in level 1 repair domain and other domains

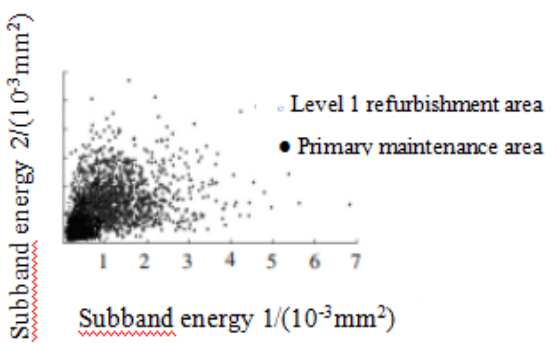

(b) Scatter distribution of control indexes in level 1 repair area - level 1 maintenance area

Figure 3. Track irregularity control index distribution corresponding to different absolute average index fields of front frame acceleration

As the region with the largest vibration response index of vehicle system components, the corresponding geometric state of the line is also the worst. It can be seen from Figure 3 (a) that the track irregularity control indexes corresponding to the level 1 repair domain are widely distributed and have certain distribution in other response domains. It can be seen from Figure 3 (b) that compared with the poor area (Level 1 repair area), the control indexes of the area with good geometric status (Level 1 repair area) gather in a small range, and are well classified with the repair area, indicating that these two control indexes play a role in domain classification.

In this paper, when SVM is used to classify the dynamic response index domain to obtain the best classification surface (i.e. domain boundary), the multi-layer 2 classification method is used to mark the joint index of track irregularity according to different dynamic response domains. The dynamic response index domain (low-level domain) with poor line status is taken as "+ 1" and the corresponding other domains are taken as "- 1". It can be seen from Figure 3 (a) that the control indexes of the repair domain and other domains are seriously overlapped. When there is no obvious category boundary between the input control indexes of different dynamic response domains, it is very difficult to use SVM algorithm to realize the automatic identification of different categories of samples. In reference [8], a method of distribution ratio of dangerous points is proposed. According to this method, this paper divides the two-dimensional distribution of control indexes into grids, calculates the ratio of the number of points NR in the low-level domain of each grid to the total number of points 
$\mathrm{n}$ in the grid, expressed by PR, which can be called the probability level value of the domain, and the calculation formula is

$$
\operatorname{Pr}=N r / N
$$

Pr represents the probability that the corresponding vehicle system component dynamic response index is in this response domain when the control index falls into this region. If PT is the threshold value, when $\operatorname{Pr}>P t$, the control indicators in this grid are all in the low-level domain (such as the renovation domain), marked with "+ 1"; when $\operatorname{Pr} \leq P t$, the scattered points in this grid are all the other joint domains corresponding to the low-level domain (such as deterioration maintenance domain), marked with "- 1". According to the requirements of the geometric state of the line, the corresponding Pt value is set. In this paper, $P t=0.7$ (that is, when $\operatorname{Pr}>0.7$, the control index in this area has a probability of at least $70 \%$ to make its corresponding dynamic response index in this area). See Figure 7 for the distribution of track irregularity control indexes in level 1 and level 2 repair areas - Level 3 maintenance areas.

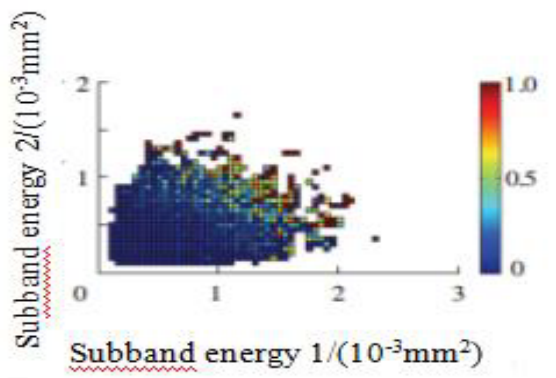

(a) Distribution of domain probability level values in repair deterioration maintenance domain

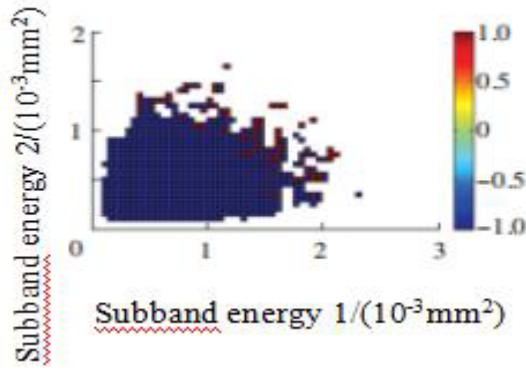

(b) Domain boundary between repair domain and deterioration maintenance domain

Figure 4. Track irregularity control index distribution of level 1 repair area and level 2 repair area - Level 3 maintenance area

It can be seen from Figure 4 that the track irregularity control index corresponding to the repair domain is basically at the position where the energy of both subbands is large, especially after the probability threshold processing (Figure 4 (b)), the classification of level 1 repair domain and other joint domains is more obvious, which reduces the difficulty of classification and recognition.

The SVM method is used to calculate the optimal classification surface between the dynamic response index domains, extract the boundary coordinates between different index domains, and perform quadratic function fitting. The domain boundaries of the absolute average acceleration index of the front frame of the vehicle system level 1 repair domain and other associated domains (Level 2 repair domain level 3 maintenance domain) are obtained. See Figure 5 for the boundary between the level 1 renovation domain and the rest of the joint domain. 


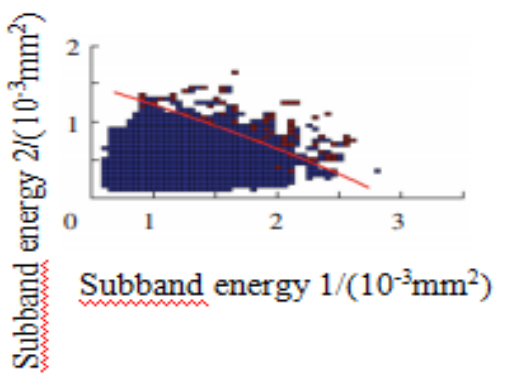

(a) Distribution of probability threshold after processing

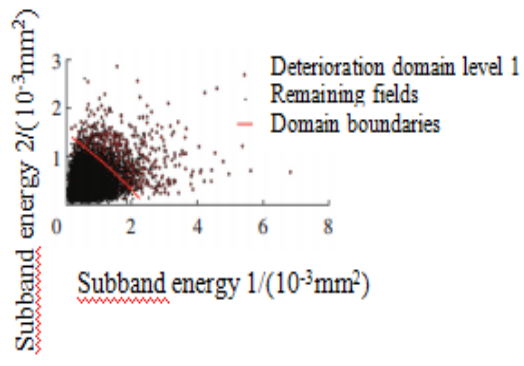

(b) Scatter distribution of initial control index

Figure 5. Domain boundary between level 1 renovation domain and other joint domains

\subsection{Boundary Estimation of Dynamic Response Index Domain}

According to the method described in Section 2.3, take $10 \mathrm{~m}$ as a calculation section of dynamic response index and track irregularity control index, estimate the boundary of dynamic response index domain for the vibration acceleration sample of front frame, and see Figure 6 for the boundary of acceleration index domain of front frame.

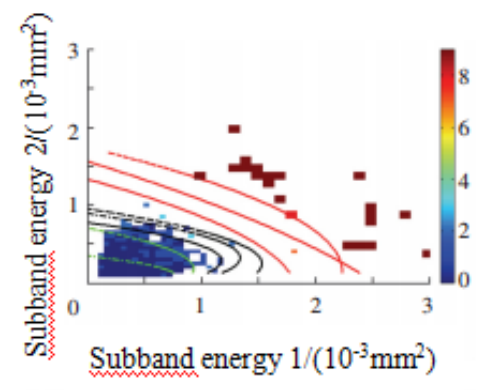

(a) Absolute average

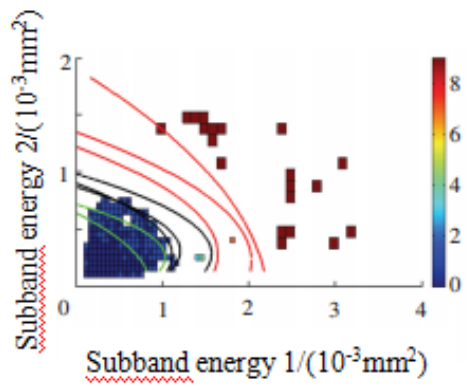

(b) Standard deviation 


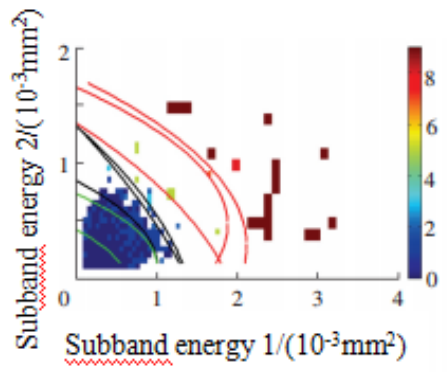

(c) Absolute maximum

Figure 6. Acceleration index domain boundary of front frame

The boundary of each domain can be expressed as a simple quadratic polynomial

$$
Y(x)=A x^{2}+B x+C
$$

Where: $\mathrm{Y}$ is Subband energy 1; $\mathrm{x}$ is subband energy 2; $\mathrm{A}, \mathrm{B}$ and $\mathrm{C}$ are polynomial coefficients.

\section{Conclusion}

By using the correlation coefficient method, two subband energy indexes are used as the control indexes of vehicle system dynamic response, and a relatively simple boundary of dynamic response index domain is established. According to the recognition accuracy of different dynamic response index fields, this method is basically feasible. Based on the basic principle of single variable method, the factors affecting the coupling of vehicle track system are divided into track irregularity and other comprehensive factors (including environment, material properties, etc.), so it can be judged to a certain extent whether the vehicle track system is abnormal and where the track system is abnormal.

\section{References:}

[1] Zhai WM. Vehicle Track Coupling Dynamics. Beijing: Science Press, 2014.

[2] Zhai WM, Zhao CF, Xia H, et al. Basic Scientific Issues on Dynamic Performance Evolution of the High-speed Railway Infrastructure and Its Service Safety. Science China Technological Sciences, 2014,44(7):645-660.

[3] Yang XG. Analysis of dynamic characteristics of high-speed railway curve. Lanzhou Jiaotong University, 2018.

[4] Pei GS. Research on harmonic geometric irregularity identification of high-speed railway based on neural network and dynamic response characteristics of vehicle system. Southwest Jiaotong University, 2018.

[5] Xu L, Chen XM. Hierarchical design and weight combination method for parameter optimization of vehicle track system. Journal of railways,2019,41(05): 177-183.

[6] Vapnik VN. The Nature of Statistical Learning Theory. New York: Springer-Verlag, 1999.

[7] Taylor JS, Cristianini N. Kernel Methods for Pattern Analysis. Cambridge: Cambridge University Press, 2004: 45-47.

[8] Lv JD. Research on local path planning method of smart cars considering vehicle dynamics. Jilin University, 2020.

[9] Xu L, Chen XM. PCA-SVM forecast of car- body vibration states of railway locomotives and vehicles under the action of track irregularity. Journal of the China Railway Society, 2014,36(7) :112-120.

[10] Qi JP. Research on reliability modeling method of high-speed railway EMUs. Lanzhou Jiaotong University, 2018. 